Reprod. Nutr. Dévelop., 1981, 21 (6A), 1015-1023.

\title{
Influence d'un entraînement physique prolongé sur la composition en acides gras du tissu adipeux épididymaire et de la carcasse chez le jeune rat soumis à un régime à base d'huile de tournesol, colza ou primor
}

\author{
par G. ROCQUELIN, P. JUANEDA
}

Station de Recherches sur la Qualité des Aliments de l'Homme I.N.R.A., 21034 Dijon Cedex, France.

Summary. Influence of physical training on the effects of dietary oils on fatty acid composition of epididymal adipose tissue and carcass lipids in the rat.

Weanling SPF male rats were fed for 12 weeks purified diets containing by weight 15 p. 100 sunflower oil, high erucic acid rapeseed oil or low erucic acid rapeseed oil. During the same period of time they were subjected to a moderate treadmill-running program and compared with sedentary animals fed the same diets. At the end of the experiment, epididymal and perirenal adipose tissues were removed and weighed, carcass lipids were extracted and weighed and their fatty acid compositions were determined by GLC on glass capillary columns.

Training decreased body weight, fat pad weight and lipid content of the carcass. However the decrease of fat pad weight in response to exercise was more important in the case of perirenal adipose tissue than in that of epididymal adipose tissue. Fatty acid composition was greatly influenced by the nature of the dietary lipids but little affected by training. Palmitic, palmitoleic, oleic, linoleic and eicosenoic acid stores in the epididymal fat of trained rats decreased but not those of stearic, linolenic and erucic acids.

\section{Introduction.}

Il est généralement admis que les tissus adipeux représentent pour l'Homme ou l'animal de laboratoire soumis à un exercice physique prolongé une source importante d'acides gras nécessaires au métabolisme énergétique des muscles et notamment du myocarde (Friedberg et al., 1963 ; Carlson, 1967 ; Masoro, 1968 ; Askew et al., 1972 ; Masoro, 1977). Il est bien connu également que la composition en acides gras de ces tissus de réserves dépend étroitement de la nature des lipides du régime, mais on ignore tout ou presque des effets de l'entraînement physique sur cette composition et surtout des interactions entre ces effets et ceux des lipides exogènes. Seule, une étude de Tsuji ef al. (1972) a montré que la composition en acides gras du tissu adipeux périrénal du rat entraîné était peu différente de celle du rat sédentaire. 
Le but de cette expérience a donc été d'examiner la composition en acides gras du tissu adipeux épididymaire et des lipides de la carcasse du rat soumis depuis le sevrage à un entraînement physique prolongé (12 semaines) et à différents régimes lipidiques. Des animaux sédentaires de même âge et ingérant les mêmes régimes ont servi de témoins. Ce travail fait partie d'une étude plus large dans laquelle on a également évalué les effets de l'entraînement physique et des huiles alimentaires sur les phospholipides d'organes (cceur, foie, muscle) ainsi que sur la morphologie et la physiologie cardiaques.

\section{Matériel et méthodes.}

Six lots de 13 rats mâles Wistar EOPS $\left({ }^{*}\right)$, provenant de l'élevage de notre laboratoire et pris au sevrage, ont été constitués ( 3 lots de rats sédentaires et 3 lots de rats entraînés). Les animaux sont mis en cages individuelles. Les rats entraînés subissent, pendant toute la durée de l'expérience (12 semaines), un entraînement physique quotidien ( 5 jours par semaine) sur tapis roulant, dont l'intensité (vitesse et durée) est progressivement augmentée au cours des 6 premières semaines (de $340 \mathrm{~m} /$ jour à la vitesse de $17 \mathrm{~m} / \mathrm{min}$, à $1100 \mathrm{~m} /$ jour à la vitesse de $22 \mathrm{~m} / \mathrm{min}$, avec une pente du tapis de $4 \mathrm{p}$. 100) puis maintenue inchangée jusqu'à la fin de l'expérience. L'épreuve a lieu l'aprèsmidi, c'est-à-dire assez loin du moment de la prise de nourriture qui se situe surtout la nuit. Les rats reçoivent un régime semi-liquide purifié dont la composition détaillée figure dans une publication antérieure (Rocquelin et Cluzan, 1968) et qui contient 15 p. 100 en poids de l'une des 3 huiles suivantes : tournesol ( $T$ ), colza riche en acide érucique $(C)$, colza (primor) pauvre en acide érucique $(P)$. La composition en acides gras des huiles est donnée dans le tableau 1.

A la fin de l'expérience et $48 \mathrm{~h}$ après le dernier entraînement, les rats sont sacrifiés par décapiłation après un jeûne de 6 à $8 \mathrm{~h}$. Les graisses épididymaires et périrénales sont immédiatement prélevées, pesées et un fragment d'environ $1 \mathrm{~g}$ de tissu épididymaire est conservé dans du chloroforme-méthanol $(2 / 1, V / V)$ et stocké à $-20^{\circ} \mathrm{C}$ jusqu'au moment des analyses. La carcasse éviscérée des animaux est congelée à $-20^{\circ} \mathrm{C}$ puis, au moment des analyses, broyée et homogénéisée à l'aide d'un broyeur à lames. Les teneurs en eau et en lipides de la carcasse sont déterminées à partir d'un échantillon de $50 \mathrm{~g}$ environ qui est lyophilisé, pesé puis hydrolysé à l'aide d'acide chlorhydrique concentré et les lipides sont extraits par l'éther de pétrole selon la technique d'Abraham ef al. (1964). L'extrait éthéré qui contient les lipides est amené à un volume connu. Une aliquote de l'extrait lipidique est prélevée, évaporée et pesée. Les lipides du tissu adipeux épididymaire sont extraits par le chloroforme-méthanol $(2 / 1, V / V)$.

Les lipides de la carcasse et du tissu adipeux sont ensuite transméthylés par le mélange benzène-trifluorure de bore-méthanol à $14 \mathrm{p} .100$ de $\mathrm{BF}_{3}(30: 70 \mathrm{~V} / \mathrm{V})$ pendant $1 \mathrm{~h} 30$ à $90^{\circ} \mathrm{C}$. Les esters méthyliques des acides gras sont dosés par chromatographie en phase gazeuse sur colonnes capillaires de verre de $25 \mathrm{~m}$ de longueur et de $0,4 \mathrm{~mm}$ de diamètre intérieur garnies de carbowax $20 \mathrm{M} \mathrm{AT}$. Nous utilisons des chromatographes équipés d'injecteurs de verre à aiguille et de détecteurs à ionisation de flamme

(*) EOPS : Exempts d’Organismes Pathogènes Spécifiques. 
TABLEAU 1

Composition (p. 100 en poids) en acides gras des lipides du régime

\begin{tabular}{lccr}
\hline Acides gras & $\begin{array}{c}\text { Tournesol } \\
(\mathrm{T})\end{array}$ & $\begin{array}{c}\text { Colza riche } \\
\text { en acide erucique } \\
(\mathrm{C})\end{array}$ & $\begin{array}{c}\text { Primor }\left({ }^{1}\right) \\
(\mathrm{P})\end{array}$ \\
\hline $16: 0$ & 6,5 & 3,5 & 4,8 \\
$16: 1 \mathrm{n}-7$ & $<0,1$ & 0,2 & 0,2 \\
$18: 0$ & 4,2 & 1,3 & 1,5 \\
$18: 1 \mathrm{n}-9$ & 24,7 & 15,8 & 61,0 \\
$18: 2 \mathrm{n}-6$ & 63,4 & 17,3 & 20,9 \\
$18: 3 \mathrm{n}-3$ & $<0,1$ & 6,8 & 9,1 \\
$20: 0$ & 0,2 & 0,6 & 0,4 \\
$20: 1 \mathrm{n}-9$ & $<0,1$ & 9,3 & 1,2 \\
$22: 0$ & 0,7 & 0,5 & 0,4 \\
$22: 1 \mathrm{n}-9$ & - & 42,9 & 0,3 \\
$24: 0$ & $<0,1$ & 0,1 & $<0,1$ \\
$24: 1 \mathrm{n}-9$ & - & 0,6 & $<0,1$ \\
\hline
\end{tabular}

(') Primor = huile de colza pauvre en acide érucique extraite d'une variété de graines françaises (Primor).

d'hydrogène. Le gaz vecteur est l'hélium. L'identification et le calcul des surfaces des pics d'acides gras sont effectués par un calculateur-intégrateur.

Les données collectées ont fait l'objet d'une analyse de variances et les moyennes ont été comparées en utilisant le test $F$ de Fisher-Snedecor avec la méthode des contrastes (Dagnélie, 1969). Cette expérience qui comprend 6 lots expérimentaux (T, $C, P$, correspondant aux 3 lots de rats sédentaires soumis aux régimes tournesol, colza et primor, et TE, CE, $P E$ correspondant aux 3 lots de rats recevant les mêmes régimes mais soumis à un entraînement physique) nous a permis de dégager les contrastes suivants : effet principal de l'entraînement physique (T, $C, P$ comparés à $T E, C E, P E)$, effet principal du type d'huile (T, TE comparés à $C, C E, P, P E)$, effet principal dû à la présence ou à l'absence d'acide érucique dans les huiles de colza (C, CE comparés à $P, P E)$, interaction type d'huile $x$ entrainement physique (T, PE, CE comparés à $T E, P$, $C)$ et interaction acide érucique $x$ entraînement physique (C, PE comparés à $P, C E$ ). Pour certains acides gras (C $18: 3$, C $20: 1$, C $22: 1$ par exemple) qui n'apparaissent dans les tissus que s'ils sont présents dans les régimes, les comparaisons n'ont été faites qu'entre les lots dans lesquels on retrouvait ces acides gras.

\section{Résultats.}

a) Carcasse (fabl. 2). - L'entraînement physique provoque un abaissement de la teneur en lipides (exprimée en $\mathrm{g} / 100 \mathrm{~g}$ de matière sèche) et une augmentation de la teneur en eau de la carcasse. La nature des huiles du régime est sans effet sur ces 2 paramètres. 


\begin{tabular}{|c|c|c|c|c|c|c|c|c|}
\hline \multirow{14}{*}{ 嵩 } & \multirow{6}{*}{ 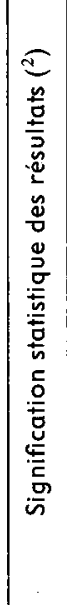 } & \multirow{2}{*}{ 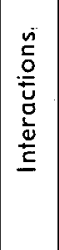 } & $\begin{array}{l}\widetilde{U} \\
x \\
w \\
\end{array}$ & $\stackrel{n}{z}$ & $\frac{N}{Z}$ & & 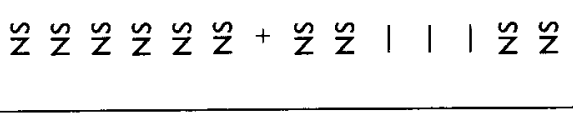 & \\
\hline & & & 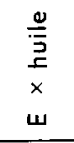 & $\stackrel{n}{Z}$ & $\tilde{z}$ & & $\tilde{z} \tilde{z} \tilde{z}+\tilde{z} \tilde{z}|| \frac{n}{z}||||$ & \\
\hline & & $\begin{array}{l}x \\
\vec{z} \\
g \\
g \\
\end{array}$ & $\overline{\check{U}}$ & $\tilde{z}$ & $\tilde{z}$ & & 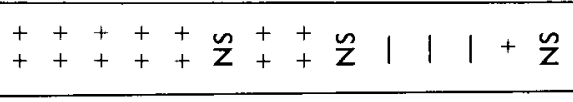 & \\
\hline & & 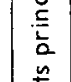 & $\frac{0}{\bar{T}}$ & $\tilde{\mathbf{z}}$ & $\stackrel{n}{Z}$ & & $\begin{array}{l}+++++1|+1| 1|1| \\
+++++1 \mid+1\end{array}$ & \\
\hline & & 岦 & ш & + & $\begin{array}{l}+ \\
+\end{array}$ & & $+\tilde{z}+++n+n+n+1 \underline{z} \tilde{z} \underline{n}+$ & \\
\hline & & & $\frac{n}{\bar{E}}$ & + & $\begin{array}{l}+ \\
+\end{array}$ & & 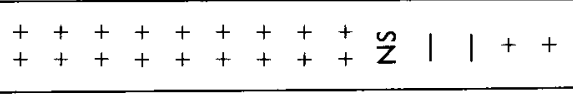 & $\frac{\vec{g}}{\frac{0}{0}}$ \\
\hline & & 는 & & ó & $m_{0}^{m}$ & & 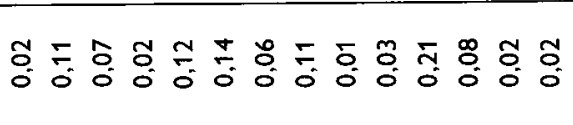 & 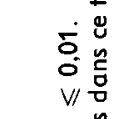 \\
\hline & & & 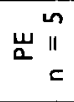 & 市 & $\begin{array}{l}\Delta \\
\dot{m}\end{array}$ & & 范 范 & 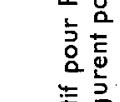 \\
\hline & & & 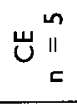 & $\hat{j}$ & นี & & 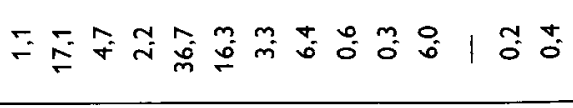 & 尊它 \\
\hline & & $E_{\bar{y}}$ & $\stackrel{\sim n}{\rightleftarrows} \underset{c}{\|}$ & fo & $\begin{array}{l}0 \\
\text { L }\end{array}$ & & 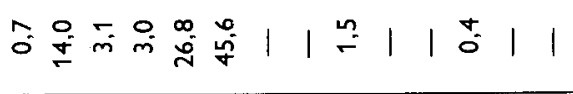 & 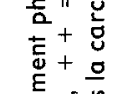 \\
\hline & & $\frac{5}{0}$ & $\therefore \stackrel{\text { in }}{\|}$ & $\stackrel{\infty}{\infty}$ & $\bar{n}$ & & 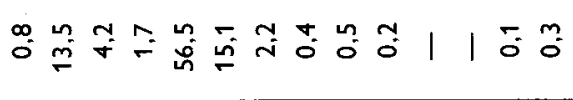 & 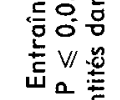 \\
\hline & & & $u \stackrel{n}{\pi}$ & $\frac{m}{i n}$ & $\bar{E}$ & & $\stackrel{0}{\circ}$ & 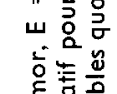 \\
\hline & & & $\vdash \stackrel{\text { in }}{\|}$ & $\stackrel{m}{q}$ & $\begin{array}{l}m \\
0 \\
\text { in }\end{array}$ & & 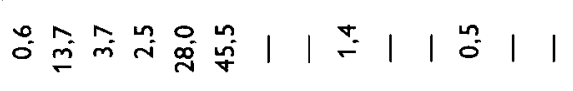 & 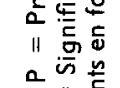 \\
\hline & & & & 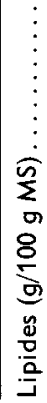 & $\begin{array}{l}\dot{\delta} \\
\dot{8} \\
\dot{0} \\
\dot{\vec{b}}\end{array}$ & 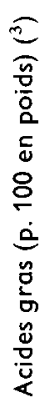 & 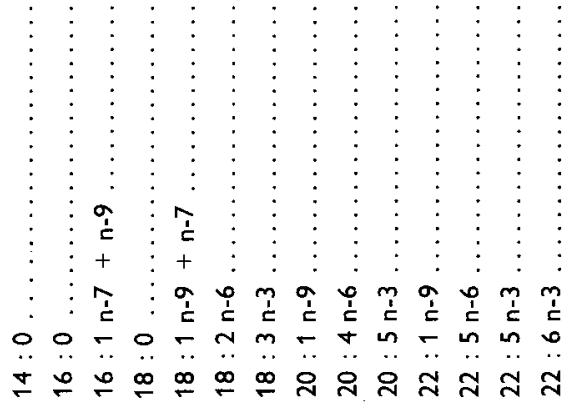 & 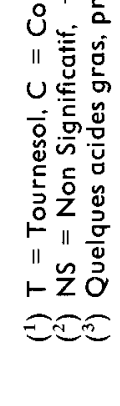 \\
\hline
\end{tabular}




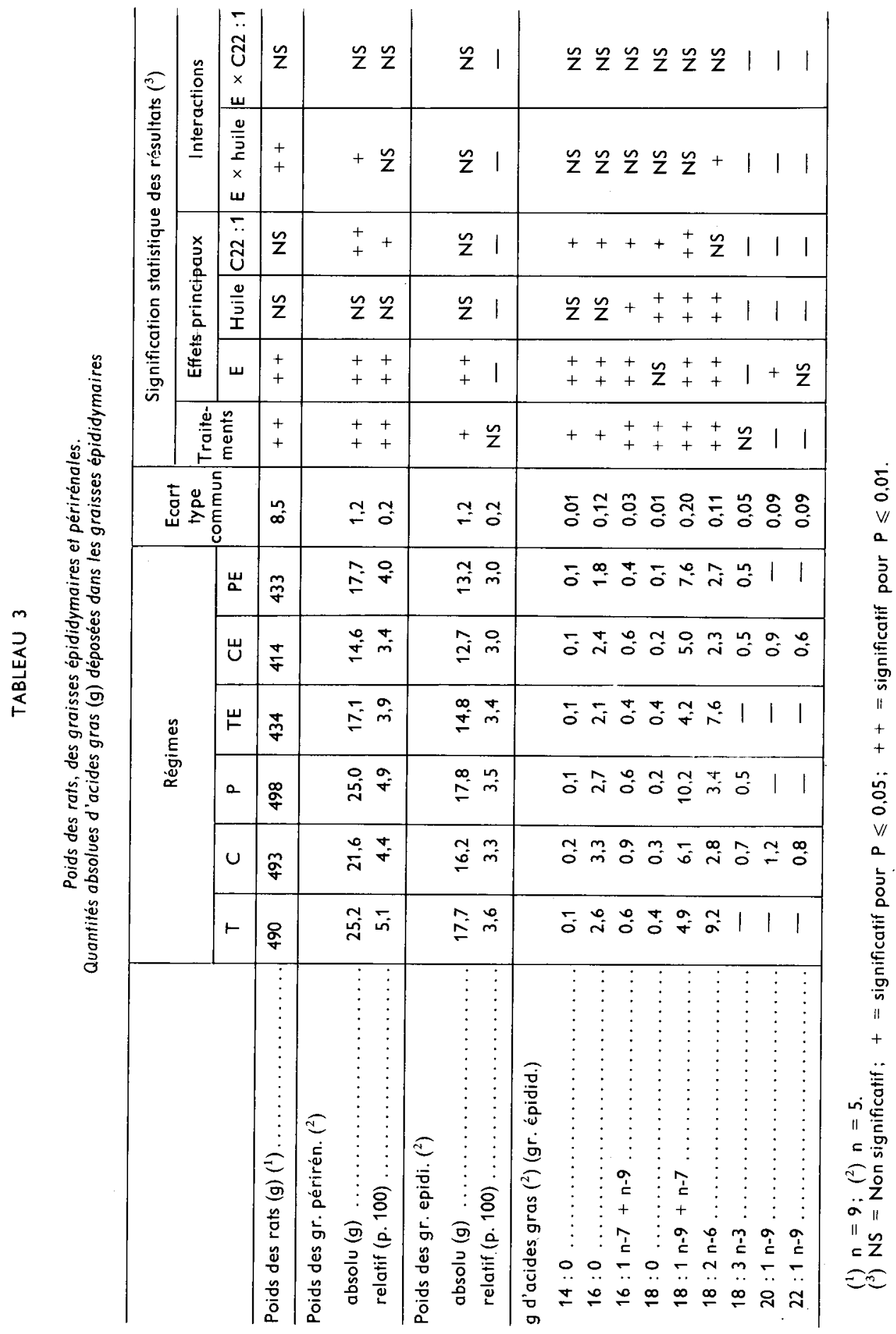


La composition en acides gras de la carcasse est étroitement liée à la nature des lipides du régime. Ainsi, la teneur en acide linoléique (C $18: 2 n-6)$ est élevée chez les rats ingérant de l'huile de tournesol alors que les monoènes $n-9$ (C $18: 1, C 20: 1$ ou $C 22: 1)$ sont prédominants chez les rats soumis aux régimes « colza » ou «primor ». Chez ces derniers également, on constate une légère incorporation de l'acide linolénique (C $18: 3 n$-3) et de ses métabolites supérieurs ( $C 20: 5, C 22: 5$ et $C 22: 6$ ). Toutefois, l'incorporation de C $18: 3$ n-3 est plus forte chez les rats ingérant de l'huile de colza bien que cette huile apporte moins d'acide linolénique que l'huile de Primor (voir tabl. 1). On retrouve plus d'acide stéarique (C $18: 0)$ chez les rats « tournesol » mais plus d'acide palmitique $(C 16: 0)$ chez les rats « colza » bien que, chez ces derniers, le régime en apporte moins que les 2 autres (voir tabl. 1). Enfin, il y a apparition de $C 16: 1$ (essentiellement de $C 16: 1 \mathrm{n}-7$ ) dans la carcasse de tous les animaux mais son pourcentage est plus élevé chez les rats «primor» et surtout «colza».

L'entraînement physique n'affecte que légèrement la composition en acides gras de la carcasse. II accroît la teneur en C 18:0 (surtout chez les rats «tournesol 》), en $C 20: 4 n-6, C 22: 6 n-3$ et diminue celle en $C 16: 1 n-7+n-9, C 18: 1 n-9+n-7$ et $C 18: 3 n-3$. Il est sans effet sur l'incorporation des acides eicosénoïque (C $20: 1 n-9)$ et érucique ( $C 22: 1 \mathrm{n}-9$ ).

b) Graisses épididymaires et périrénales (tabl. 3). - Le poids des graisses épididymaires et périrénales est plus faible chez les rats entraînés. Ceci est dô, pour les graisses périrénales, à un effet réel de l'entraînement physique (poids absolu et relatif plus faibles) mais pour les graisses épididymaires seulement au fait que les animaux sont moins gros. En outre, la diminution du poids des graisses périrénales est plus forte chez les rats entraînés nourris à l'huile de tournesol que chez les autres animaux.

La composition en acides gras des graisses épididymaires a été exprimée, pour chaque acide gras, en valeur absolve ( $g$ dans la totalité du tissu) pour tenir compte des variations importantes du poids des dépôts adipeux. On constate que la nature des acides gras stockés comme celle des acides gras de la carcasse est liée en grande partie aux apports lipidiques exogènes. Ainsi, on trouve surtout du $C 18: 2$ n-6 chez les rats «tournesol » et des monoènes $n-9$ chez les rats « colza » et « primor ». Le C $18: 3 n-3$ apparaît dans les dépôts adipeux des rats qui en ingèrent (rats « colza » et « primor ») et le C $18: 0$ s'incorpore en plus forte quantité chez les rats «tournesol ». Quant aux teneurs en $C 16: 0$ et $C 16: 1$ (essentiellement $C 16: 1 n-7$ ), beaucoup moins liées aux apports exogènes, elles sont plus élevées chez les rats «colza ».

L'entraînement physique provoque une nette diminution des réserves du tissu en C $14: 0$, C $16: 0$, C $16: 1 n-7+n-9$, C $18: 1 n-9+n-7$, C $18: 2 n-6$, C $20: 1 n-9$, mais il est sans effet sur la teneur en $C 18: 0, C 18: 3 n-3$ et $C 22: 1 n-9$. Par ailleurs, la nature de l'huile du régime n'a que peu d'incidence sur les effets de l'entraînement physique. On constate seulement que la disparition du $C 18: 2$ n-6 est plus marquée chez les rats entraînés ingérant de l'huile de tournesol.

\section{Discussion.}

De très nombreux travaux ont montré que la composition en acides gras des réserves adipeuses du rat dépendait largement de la nature des acides gras exogènes. 
En ce qui concerne plus spécifiquement l'influence des huiles de colza et de primor, nous confirmons globalement les données acquises antérieurement chez le rat sédentaire, que ce soit sur la carcasse (Beare, 1961 ; Beare ef al., 1963 ; Craig et al., 1963 ; Craig et Beare, 1968 ; Hornstra, 1972) ou sur les graisses épididymaires (Craig ef al., 1963 ; Rocquelin et al., 1970 ; Ziemlanski et al., 1973 ; Rocquelin et Juaneda, 1976). La composition en acides gras de ces 2 prélèvements est d'ailleurs sensiblement la même sauf toutefois en ce qui concerne les acides gras polyinsaturés en $C 20$ et $C 22$ $n-6$ ou $n-3$, que l'on trouve dans les lipides de la carcasse, mais qui ne sont pas des acides gras de réserves car ils proviennent des phospholipides des muscles et du cerveau.

Nous confirmons également que les teneurs en $C 16: 0, C 16: 1$ (n-7 essentiellement) et $C 18$ : 0 des tissus adipeux sont beaucoup moins liées aux apports exogènes que ne le sont celles en acides insaturés à 18,20 ou 22 atomes de carbone. Le C $16: 0$, provient d'une biosynthèse endogène, importante dans le tissu adipeux, et il sert de précurseur au C 16:1 n-7 et au C $18: 0$ (Shorland, 1955). II semblerait toutefois, d'après nos résultats et ceux de Craig et al. (1963) que cette biosynthèse soit plus intense dans les dépôts adipeux des rats ingérant de l'huile de colza.

Nous avons aussi retrouvé, comme beaucoup d'autres auteurs (Jones ef al., 1964 ; Hanson ef al., 1967 ; Crews ef al., 1969 ; Askew ef al., 1972 ; Askew et al., 1973 ; Parísková et Poledne, 1974 ; Askew ef al., 1975a, b ; Pitts et Bull, 1977 ; Askew ef al., 1977 ; Lau et al., 1979), une diminution sensible du poids des graisses épididymaires et périrénales due à l'entraînement physique. Ces résultats témoignent de la mobilisation accrue des acides gras des tissus adipeux vers les muscles dont le métabolisme énergétique, au cours d'un exercice physique prolongé, est augmenté. Cependant, il semble ressortir de notre étude que l'activité métabolique du tissu adipeux (équilibre entre lipogenèse et lipolyse de triglycérides), varie d'un tissu adipeux à un autre ef aussi en fonction de la nature des lipides du régime puisque la baisse de poids des graisses périrénales est plus accentuée que celle des graisses épididymaires surtout chez les rats ingérant de l'huile de tournesol. Ce résultat, toutefois, ne concorde pas avec ceux d'Oscai (1979) qui donnent à penser, au contraire, que les différentes réserves adipeuses du rał entraîné ont une activité métabolique équivalente.

Il apparaît aussi dans notre étude que les teneurs des principaux acides gras du tissu adipeux épididymaire (C $16: 0, C 16: 1 n-7, C 18: 1 n-9, C 18: 2$ n-6 et C 20 : $1 \mathrm{n}-9)$ diminuent fortement au cours de l'effort. Toutefois, on a pu remarquer que les réserves de $C 18: 0, C 18: 3$ n-3 et $C 22: 1$ n-9 sont peu modifiées par l'entraînement physique. Ces acides gras sont peut-être moins bien utilisés par les muscles au travail, ou alors, leur captation ou leur mobilisation par les lipases du tissu adipeux au cours de l'effort peuvent être aussi différentes de celles des autres acides gras (C $16: 0$, C 18 : 1 n-9, C $18: 2$ n-6) ; Hülsmann et al. (1979) ont déjà montré que, chez le rat sédentaire nourri pendant 4 jours avec de l'huile de colza, l'activité de la lipoprotéinelipase du tissu adipeux épididymaire était plus forte que chez les rats témoins alors, qu'au contraire, celle de la triglycéride-lipase diminuait. ll serait donc intéressant de compléter ce travail par une mesure, dans différents tissus adipeux, de l'activité des lipases chez le rat entraîné et soumis à différents régimes lipidiques.

En conclusion de cette étude, il ressort que l'entraînement physique n'a que peu d'effet sur la composition qualitative en acides gras du tissu adipeux. Celle-ci dépend 
avant tout de la nature des lipides du régime. Toutefois, il sembleraił que l'intensité de la mobilisation des acides gras, au cours de l'entraînement physique, soit plus forte dans le tissu adipeux périrénal que dans le tissu adipeux épididymaire et qu'elle puisse aussi être différente selon la nature de l'acide gras stocké.

Reçu en mai 1981. Accepté en juin 1981.

\section{Références}

ABRAHAM J., MORIN-JOMAIN M., PERETIANU J., 1964. Nouvelle technique de détermination de la composition corporelle des animaux de laboratoire. Bull. Soc. Chim. biol., 46, 755-758.

ASKEW E. W., DOHM G. L., HUSTON R. L., SNEED T. W., DOWDY R. P., 1972. Responses of rat tissue lipases to physical training and exercise. Proc. Soc. exp. Biol. Med., 141, 123-129.

ASKEW E. W., HUSTON R. L., DOHM G. L., 1973. Effect of physical training on esterification of glycerol-3-phosphate by homogenates of liver, skeletal muscle, heart, and adipose tissue of rats. Mefabolism, 22, 473-480.

ASKEW E. W., DOHM G. L., DOUB W. H. Jr., HUSTON R. L., VAN NATTA P. A., 1975a. Lipogenesis and glyceride synthesis in the rat : Response to diet and exercise. J. Nutr., 105, 190-199.

ASKEW E. W., BARAKAT H., KUHL G. L., DOHM G. L., 1975b. Response of lipogenesis and fatty acid synthetase to physical training and exhaustive exercise in rats. Lipids, 10, 491-496.

ASKEW E. W., HECKER A. L., WISE W. R. Jr., 1977. Dietary carnitine and adipose fissue furnover rate in exercise trained rats. J. Nutr., 107, 132-142.

BEARE J. L., 1961. The influence of dietary fat on the fatty acid composition of liver, carcass and milk of rats. Can. J. Biochem. Physiol., 39, 1855-1863.

BEARE J. L., CAMPBELL J. A., YOUNGS C. G., CRAIG B. M., 1963. Effects of saturated fat in rats fed rapeseed oil. Can. J. Biochem. Physiol., 41, 605-612.

CARLSON L. A., 1967. Lipid metabolism and muscular work. Fed. Proc., 26, 1755-1759.

CRAIG B. M., BEARE J. L., 1968. Nutritional properties of canadian canbra oil. Can. Inst. Food Technol. J., 1, 64-67.

CRAIG B. M., YOUNGS C. G., BEARE J. L., CAMPBELL J. A., 1963. Fatty acid composition and glyceride structure in rats fed rapeseed oil or corn oil. Con. J. Biochem. Physiol., 41, 43-49.

CREWS E. L. III, FUGE K. W., OSCAI L. B., HOLLOSZY J. O., SHANK R. E., 1969. Weight, food intake, and body composition : effects of exercise and of protein deficiency. Am. J. Physiol., 216, 359-363.

DAGNÉLIE P., 1969. Théories ef méthodes statistiques. Les Presses agronomiques de Gembloux.

FRIEDBERG S. J., SHER P. B., BOGDONOFF M. D., ESTES E. H., 1963. The dynamics of plasma free fatty acid metabolism during exercise. J. Lip. Res., 4, 34-38.

HANSON D. L., LORENZEN J. A., MORRIS A. E., AHRENS R. A., WILSON J. E. Jr., 1967. Effects of fat intake and exercise on serum cholesterol and body composition of rats. Am. J. Physiol., 213, 347-352.

HORNSTRA G., 1972. Digestibility, efficiency and other metabolic effects of dietary rapeseed oil in rats. Nutr. Metabol., 14, 282-297.

HÜLSMANN W. C., GEELHOED-MIERAS M. M., JANSEN H., HOUTSMULLER U. M. T., 1979. Alteration of the lipase activities of muscle, adipose tissue and liver by rapeseed oil feeding of rats. Biochim. biophys. Acta, 572, 183-187.

JONES E. M., MONTOYE H. J., JOHNSON P. B., Sister MARTIN J. M., VAN HUSS W. D., CE DERQUIST D., 1964. Effect of exercise and food restriction on serum cholesterol and liver lipids. Am. J. Physiol., 207, 460-466.

LAU H. C., FLAIM E., RITCHEY S. J., 1979. Body weight and depot fat changes as influenced by exercise and dietary fat sources in adult BHE rats. J. Nufr., 109, 495-500.

MASORO E. J., 1968. Lipid metabolism during exercise, 222-228. In MASORO E. J., Physiological chemistry of lipids in mammals, W. B. Saunders Company.

MASORO E. J., 1977. Lipids and lipid metabolism. Ann. Rev. Physiol., 39, 301-321. 
OSCAI L. B., 1979. Effect of acute exercise on tissue free fatty acids in untrained rats. Can. J. Physiol. Pharmacol., 57, 485-489.

PARIZKOVA J., POLEDNE R., 1974. Consequences of long-term hypokinesia as compared to mild exercise in lipid metabolism of the heart, skeletal muscle and adipose tissue. Europ. J. appl. Physiol., 33, 331-338.

PITTS G. C., BULL L. S., 1977. Exercise dietary obesity and growth in the rat. Am. J. Physiol., 232, R38-R34.

ROCQUELIN G., CLUZAN R., 1968. L'huile de colza riche en acide érucique et l'huile de colza sans acide érucique : valeur nutritionnelle ef effets physiologiques chez le rat. Ann. Biol. anim. Bioch. Biophys., 8, 395-406.

ROCQUELIN G., JUANEDA P., 1976. Incorporation comparée à très court terme, des acides n-9 trans-docosénoïque (brassidique) et n-9 cis-docosénoïque (érucique) dans le tissu adipeux du rat. C. R. Soc. Biol., 170, 1051-1056.

ROCQUELIN G., MARTIN B., CLUZAN R., 1970. Comparative physiological effects of rapeseed and canbra oils in the rat : influence of the ratio of saturated to monounsaturated fatty acids. Proc. int. Conf. Sci. Technol. Market. of rapeseed products, Ste Adèle, Québec, 405422.

SHORLAND F. B., 1955. Formation of animal fats, 275-325. In HOLMAN R. T., LUNDBERG W. O., MALKIN T., Progress in the chemistry of fats and other lipids, Vol. 3. Pergamon Press.

TSUJI E., OSHIMA S., SUZUKI S., 1972. Effect of nutrition and physical exercise on fatty acid composition of adipose tissue in rats. Jap. J. Nutr., 30, 53-57.

ZIEMLANSKI S., KUCHARCZYK B., BULHAK-JACHYMCZYK B., 1973. The effect of selected edible fats on fatty acid composition of tissue lipids. Pol. med. Sci. H. Bull., 15, 563-576. 